\title{
Dennis Schulting* Probleme des ,kantianischen' Nonkonzeptualismus im Hinblick auf die B-Deduktion
}

DOI 10.1515/kant-2015-0052

\begin{abstract}
Recently, Allais, Hanna and others have argued that Kant is a nonconceptualist about intuition and that intuitions refer objectively, independently of the functions of the understanding. Kantian conceptualists have responded (e.g. with reference to $\mathrm{KrV}$, A $89 \mathrm{ff} . / \mathrm{B} 122 \mathrm{ff}$. (§13), which the nonconceptualists also cite as textual evidence for their reading) that this view conflicts with the central goal of Kant's Transcendental Deduction: to argue that all intuitions are subject to the categories. I argue that the conceptualist reading of $\mathrm{KrV}$, A $89 \mathrm{ff}$./B $122 \mathrm{ff}$. is unfounded. Further, I argue that the nonconceptualists are wrong to believe that intuitions as such refer objectively and that they are mistaken about the relation between figurative synthesis and intellectual synthesis.
\end{abstract}

Keywords: Nonkonzeptualismus, Transzendentale Deduktion, figürliche Synthesis.

Eines der großen Probleme beim Versuch, Kants ,Transzendentale Deduktion der Kategorien' zu verstehen, betrifft die Frage nach dem, was Dieter Henrich in einer bekannten, 1969 erstmals auf Englisch erschienenen Abhandlung „the second step“ der Argumentation der B-Deduktion genannt hat. ${ }^{1}$ Der von Henrich unterbreitete Vorschlag einer zweistufigen Struktur der B-Deduktion ist allgemein akzeptiert worden, aber Einigkeit über die genaue Interpretation dessen, was im

Dieser Aufsatz beruht auf einem Vortrag, den ich in einem von 16. bis 18. Oktober 2013 in Mainz abgehaltenen Seminar über „Kants ,Transzendentale Deduktion“ in der B-Fassung“ vorgetragen habe. Ich bedanke mich bei Heiner F. Klemme und Falk Wunderlich für die Einladung zur Teilnahme an der Tagung. Ich möchte zudem Wolfgang Ertl, Giuseppe Motta, Christian Onof, Marcel Quarfood, Scott Stapleford und Jacco Verburgt für ihre schriftlichen Bemerkungen zu einer früheren Fassung dieses Aufsatzes danken, und ich danke Wolfgang Ertl überdies für seine Überprüfung des deutschen Textes.

1 Henrich, Dieter: „The Proof-Structure of Kant's Transcendental Deduction“. In: Review of Metaphysics xxii (4) (1969): 640-659. Deutsch in: Kant. Zur Deutung seiner Theorie von Erkennen und Handeln. Hrsg. von G. Prauss. Köln 1973, 90-104.

*Kontakt: Dr. Dennis Schulting: dennis.schulting@t-online.de 
sogenannten ,zweiten Schritt‘ eigentlich bewiesen werden soll, besteht noch immer nicht. In groben Umrissen gesagt, legt Kant in diesem ,zweiten Schritt` die Möglichkeit nicht nur der Objekte überhaupt und deren Gedachtwerdens, was im ,ersten Schritt' der B-Deduktion schon erwiesen worden sein soll, sondern auch der Erkenntnis von empirischen raumzeitlichen Objekten dar.

Ich möchte mich hier jedoch nicht mit dieser anhaltenden Debatte beschäftigen, sondern mit der Frage, inwieweit bestimmte Probleme einer jüngst begonnenen Debatte über Kants vermeintlichen ,Nonkonzeptualismus“ mit den durch Henrichs Aufsatz aufgeworfenen Fragen nach der Beweisstruktur der B-Deduktion zusammenhängen. Mein Interesse gilt diesem ,kantianischen“ Nonkonzeptualismus als Interpretationsmodell der Deduktion. Kurzum, es geht hier um die Frage, inwiefern dieses Interpretationsmodell von Nutzen sein kann, um Kants Beweisverfahren in der Analyse wenigstens ansatzweise nachzuvollziehen. Im Kontext der Nonkonzeptualismus-Debatte stellt sich die zentrale Frage: „Beabsichtigt Kant zu beweisen, dass alle Anschauungen bzw. Erscheinungen überhaupt von den Funktionen des Denkens abhängen?“2 Dies wird von den Nonkonzeptualisten grundsätzlich verneint, während die Konzeptualisten diese Frage bejahen.

Im Rahmen meines Aufsatzes möchte ich dies wie folgt zuspitzen. Die Nonkonzeptualismus-Debatte hat gewissermaßen ein interpretatives Paradoxon hervorgebracht, welches sie nicht imstande ist zu lösen. Das heißt, sowohl Konzeptualisten wie Nonkonzeptualisten behaupten, die Beweisführung in der Deduktion zeige, dass Kant hauptsächlich ein Konzeptualist bzw. Nonkonzeptualist, oder wie einige Nonkonzeptualisten meinen, dass Kant sogar beides sei. ${ }^{3}$ Dass er beides ist, kann aber logischerweise nicht der Fall sein, wenn Konzeptualismus und Nonkonzeptualismus gerade diametral konträren Standpunkte darstellen, oder Kant müsste sich widersprechen. Letzten Endes muss man sich ernsthaft die Frage stellen, ob diese Debatte überhaupt zum besseren Verständnis der Deduktion beizutragen vermag und nicht vielmehr die Kernfrage der Deduktion, nämlich die Frage, wie Anschauung und Begriffe tatsächlich als auf einander

2 Vgl. KrV, B 136 und besonders die Konklusion des, ersten Schrittes ' der B-Deduktion in B 143. Die Beweisführung des ,zweiten Schrittes ' in B 159 f., in $\S 26$ der B-Deduktion, wo Kant zu behaupten scheint, sogar „alles, was unseren Sinnen nur vorkommen mag“, sei notwendig subsumierbar unter den Kategorien, behandle ich ausführlich in Schulting, Dennis: Kant's Radical Subjectivism. Perspectives on the Transcendental Deduction. London und New York (im Erscheinen), Kap. 7.

3 Siehe Hanna, Robert: „Kantian Non-Conceptualism“. In: Philosophical Studies 137, 2008, 41-64, hier 45 und ders.: „Kant, Hegel, and the Fate of Non-Conceptual Content“. In: Hegel Bulletin 34 (1), Nr. 67, 2013, 1-32, hier 13. 
bezogen betrachtet werden müssen, wegen dieses gegensätzlichen theoretischen Ausgangspunktes (entweder Nonkonzeptualismus oder Konzeptualismus) grundlegend verzerrt.

\section{Die nonkonzeptualistische Kantinterpretation gegenüber dem ,kantianischen' Konzeptualismus}

Zuerst möchte ich einige kantische Begriffsdefinitionen kurz in Erinnerung rufen sowie definieren, was unter ,Konzeptualismus“ bzw. ,Nonkonzeptualismus‘, und zwar besonders im kantischen Kontext, verstanden wird.

Kant definiert „Anschauung“ als „eine objective Perception“, die „sich unmittelbar auf den Gegenstand [bezieht]“ (KrV, B 376 f./A 320), „Wahrnehmung“ als eine „mit Empfindung begleitete Vorstellung“ (KrV, B 147) und „Erscheinung“ als den ,unbestimmte[n] Gegenstand einer empirischen Anschauung“ (KrV, B 34/A 20). Diese Begriffe sind offensichtlich nicht äquivalent, aber in der Nonkonzeptualismus-Debatte werden sie leider manchmal so verwendet, als wären sie es. Ich werde darauf in diesem Aufsatz allerdings nicht näher eingehen.

Robert Hanna ${ }^{5}$ zufolge kann ,Konzeptualismus‘ knapp definiert werden als die These, dass (1) jedweder bewusste, objektive Vorstellungsinhalt, jedwede Anschauung bzw. jede Erscheinung vom diskursiven Begriffsvermögen bestimmt wird, d.h., dass Anschauungen uns nur Objekte liefern können, wenn sie unter Begriff gebracht worden sind, und dass (2) nicht-menschliche Wesen (d.h. Tiere) nicht über ein derartiges Begriffsvermögen verfügen, mithin keine Objekte im strengen Sinne anschauen bzw. wahrnehmen können.

Der ,Nonkonzeptualismus“ vertritt dagegen die These, dass (1) nicht jeder bewusste, objektive Vorstellungsinhalt lediglich oder überhaupt vom Begriffsvermögen bestimmt wird und dass Anschauungen uns unabhängig von Begriffen

4 Vgl. V-Met/Schön, AA 28: 482: „Die Vorstellung insofern mir dadurch ein Objekt unmittelbar gegeben wird, nennt man Anschauungen“; und ebd., AA 28: 484: „Unter einer Anschauung versteht man nicht nur eine Vorstellung des Sehens; sie bedeutet jede unmittelbare Vorstellung eines Gegenstandes so fern er gegeben ist.“ In Prol, AA 04: 281 definiert Kant eine Anschauung als „eine Vorstellung, so wie sie unmittelbar von der Gegenwart des Gegenstandes abhängen würde“, was neben den Singularitäts- und Unmittelbarkeitskriterien der Anschauung noch ein drittes Kriterium der Abhängigkeit suggeriert (vgl. KrV, B 72).

5 Hanna, Robert: „The Togetherness Principle, Kant’s Conceptualism, and Kant's Non-Conceptualism“. In: Stanford Encyclopedia of Philosophy, http://plato.stanford.edu/archives/fall2013/ entries/kant-judgment/supplement1.html, abgerufen am 13. Dezember 2013. 
Objekte unmittelbar liefern oder „präsentieren“6 können, (2) mindestens einige vernünftige, menschliche, objektive Bewusstseinsinhalte unabhängig vom Begriffsvermögen vorkommen und nicht vom letzteren bestimmt sind, und dass (3) mindestens bei einigen nicht-menschlichen Wesen (d.h. Tieren) ein objektiver Bewusstseinsinhalt vorliegt und sie sich nicht lediglich (quasi-)mechanisch zu ihrem Umwelt verhalten, d.h., dass sie wirkliche Gegenstände irgendwie anschauen bzw. wahrnehmen können. ${ }^{7}$

6 Allais, Lucy: „Kant, Non-Conceptual Content and the Representation of Space“. In: Journal of the History of Philosophy 47 (3), 2009, 383-413, hier 384, 386. Allais vertritt die Ansicht, der Raum sei „a condition of the possibility of being perceptually presented with outer particulars“ (ebd., 384; Hervorhebung von mir), d. h., ,intuitions represent objects immediately because they present the particular object itself, as opposed to being representations that enable us to think about it whether it is present or not“ (ebd., 389). Der (starke) Konzeptualismus sei gerade die entgegengesetzte Ansicht, nämlich ,that we cannot be perceptually presented with a particular independently of the application of concepts“ (ebd., 386).

7 Ich möchte hier den in der Literatur üblichen Unterschied zwischen dem ,Inhalts-Nonkonzeptualismus' (,content nonconceptualism) und dem ,Zustands-Nonkonzeptualismus‘ (,state nonconceptualism`) übergehen. Der ,Inhalts-Nonkonzeptualismus‘ besagt, dass der Inhalt von Anschauungen und der Inhalt von begrifflichen Äußerungen der Art nach verschieden sind, so dass der Anschauungsinhalt unseres Begriffsvermögen grundsätzlich übersteigt, während der ,Zustands-Nonkonzeptualismus‘ besagen will, dass, obwohl Anschauungen und begriffliche Äußerungen denselben Inhalt aufweisen, ein Subjekt sich in einem Zustand befinden kann, in dem es nicht über Begriffe verfügt, mithilfe deren es Aussagen über den Inhalt seiner Anschauungen machen könnte. Dieser Unterschied scheint für die einschlägige kantische Frage nach der beanspruchten Abhängigkeit der Anschauungen von den Funktionen des Denkens irrelevant zu sein, denn letzteres bezieht sich gerade auf die mögliche Relation zwischen Anschauungen und a priori-Begriffen, die mit der These vom ,Inhalts-Nonkonzeptualismus' schon begrifflich ausgeschlossen zu sein scheint. Andererseits scheint der ,Zustands-Nonkonzeptualismus‘ zu implizieren, dass, obwohl ein Subjekt sich in einem Zustand befinden könnte, in dem es nur Anschauungen hat ohne über Begriffe zu verfügen bzw. verfügen zu können, damit es diese Anschauungen beschreiben könnte, die Bewusstseinsinhalte selbst immer schon implizit begrifflich sind. Deswegen könnte man meinen, der ,Zustands-Nonkonzeptualismus‘ sei nur im sehr groben Sinne als ,nonkonzeptualistisch“ zu bezeichnen (vgl. Hanna: „Kant, Hegel, and the Fate of Non-Conceptual Content“, op. cit., 5). Hanna zufolge ist der ,Inhalts-Nonkonzeptualismus“ deshalb die einzig legitime Form des Nonkonzeptualismus. Damit man aber vermeiden kann, die oben erwähnte radikale Konsequenz aus dem ,Inhalts-Nonkonzeptualismus‘ ziehen zu müssen, wird ein Unterschied zwischen dem ,absoluten` oder ,essentialistischen` Nonkonzeptualismus und dem ,relativen“ oder ,nicht-essentialistischen` Nonkonzeptualismus gemacht (Allais, op. cit., 386; Hanna: „The Togetherness Principle, Kant’s Conceptualism, and Kant’s Non-Conceptualism“, op. cit., unterscheidet zwischen ,starkem‘ bzw. ,schwachem‘ Nonkonzeptualismus). Der ,relative‘ Nonkonzeptualismus lässt ausdrücklich die Möglichkeit offen, dass ungeachtet des wesentlichen Unterschiedes zwischen Anschauungsinhalt und Begriffsinhalt der Inhalt von Anschauungen in begrifflichen Aussagen artikuliert wird. Siehe weiter unten Fußnote 19. 
Wenn nun die konzeptualistische These in den explizit kantischen Kontext übertragen wird, dann sollte man ergänzen, dass der Verstand, also das Begriffsvermögen (KrV, A 68f./B 93f.), nicht nur der a priori-Bestimmungsgrund jedes kognitiven Urteils, sondern auch der der Sinnlichkeit selbst ist (,starker Konzeptualismus'); oder, in abgeschwächter Form wird die These vertreten, dass, obwohl der Verstand alle kognitiven Urteile - inklusive empirischer Anschauungen - bestimmt, die Sinnlichkeit jedoch unabhängig vom Verstand eine notwendige Bedingung des objektiv-bewussten Vorstellungsinhaltes, der Anschauung bzw. der Erscheinung ist und etwaige begriffliche,Aktivitäten‘ (z. B. demonstrative Bezugnahme) nicht notwendigerweise innerhalb des explizit vom Verstand begründeten kognitiven Urteils stattfinden müssen (,schwacher Konzeptualismus').

Für beide Positionen, den ,starken“ wie den ,schwachen' Konzeptualismus, gilt, dass Anschauungen keinen wesentlich anderen Vorstellungsinhalt aufweisen als begriffliche Vorstellungen. Ihr Unterschied liegt hauptsächlich darin, dass der ,starke‘ Konzeptualismus die von Kant mehrmals betonte strenge Trennung zwischen Verstand und Sinnlichkeit dermaßen aufgeweicht hat, dass eine fast völlige Verschmelzung beider droht, welche der ,schwache‘ Konzeptualismus gerade vermeiden möchte. Man könnte sich nun auf den Standpunkt stellen - und genau dies tun die meisten ,kantianischen' Konzeptualisten wie Robert Pippin, John McDowell, Christian Wenzel und Hannah Ginsborg ${ }^{8}$-, Kant sei grundsätzlich ein Konzeptualist im schwachen Sinn, da es in seiner Erkenntnistheorie

8 Pippin, Robert: „Hegel's Original Insight“. In: International Philosophical Quarterly xxxiii (3), 1993, 285-295; ders.: „Concept and Intuition: On Distinguishability and Separability“. In: Hegel-Studien 39/40, 2005, 25-39; ders.: „What is Conceptual Activity?“. In: Mind, Reason, and Being-in-the-World. The McDowell-Dreyfus Debate. Hrsg. von J. K. Schear. London und New York 2013, 91-109. McDowell, John: Mind and World, 2. Auflage. Cambridge/MA 1996; ders.: Having the World in View: Essays on Kant, Hegel, and Sellars. Cambridge/MA 2009. Wenzel, Christian: „Spielen nach Kant die Kategorien schon bei der Wahrnehmung eine Rolle? Peter Rohs und John McDowell“. In: Kant-Studien 96, 2005, 407-426. Ginsborg, Hannah: „Was Kant a Nonconceptualist?“. In: Philosophical Studies 137, 2008, 65-77. Siehe auch Land, Thomas: „Kantian Conceptualism“. In: Rethinking Epistemology. Hrsg. von G. Abel und J. Conant. Berlin und New York 2011, 197-239; Bauer, Nathan: „A Peculiar Intuition: Kant’s Conceptualist Account of Perception“. In: Inquiry 55 (3), 2012, 215-237; Griffith, Aaron: „Perception and the Categories: A Conceptualist Reading of Kant's Critique of Pure Reason“. In: European Journal of Philosophy 20 (2), 2012, 193222; Williams, Jessica: „How Conceptually Guided are Kantian Intuitions?“ In: History of Philosophy Quarterly 29 (1), 2012, 57-78 und Gomes, Anil: „Kant on Perception: Naïve Realism, NonConceptualism, and the B-Deduction“. In: The Philosophical Quarterly 64 (254), 2014, 1-19. Für eine Auseinandersetzung mit McDowells Lesart siehe Schulting, Dennis: „Kant, Non-Conceptual Content, and the ,Second Step“ of the B-Deduction“. In: Kant Studies Online, 2012, 51-92. Ich behandle Pippin ausführlich im Kontext seiner Deutung der Hegelschen Kant-Kritik in Schulting: Kant's Radical Subjectivism, op. cit., Kap. 8. 
heißt, und zwar laut des berühmten Passus in $\mathrm{KrV}$, B 75/A 51 ${ }^{9}$, dass Begriffe und Anschauungen ungeachtet ihres formalen Unterschiedes immer im Zusammenhang vorkommen müssen. Kants Grundthese von der Differenz von Anschauung und Begriff könne mithin nicht so gelesen werden, als ob Anschauungen irgendwie vorliegen könnten, ohne dass Begriffe oder ,begriffliche Aktivität‘ in gewisser Hinsicht involviert wären. McDowell drückt dies so aus: Anschauungen liefern keinen „denkbaren unterschiedlichen Beitrag“ zur Erkenntnis ${ }^{10}$. Der „Erkenntnisvollzug“ könne deshalb nicht „,in zwei Faktoren“ aufgespalten werden, ${ }^{11}$ wobei die Beziehung zwischen Anschauungen und den Kategorien erst nachträglich, etwa exogen, herangetragen werde. ${ }^{12}$ Anders formuliert: Anschauungen sind, so verstanden, nicht wirklich unabhängig von oder ,selbständig' mit Bezug zu Begriffen, ${ }^{13}$ oder wenn sie auf solche Weise existierten, dann wären sie völlig bedeutungslos (d. h. wenigstens für uns).

Nonkonzeptualisten wie Hanna ${ }^{14}$ und Lucy Allais ${ }^{15}$ behaupten hingegen, mehrere Textstellen in der Kritik der reinen Vernunft belegten, dass Kant eher die reale Unabhängigkeit der Anschauungen von den Funktionen des Verstandes unterstreiche, am deutlichsten in KrV, A 89 f./B 122f., d. h. im einleitenden Paragraphen 13 der ,Transzendentalen Deduktion“ (siehe u. a. auch B 145). Ich werde diesen Passus im Paragraphen 13 weiter unten eingehend behandeln.

Dieser nonkonzeptualistischen Ansicht zufolge sind Anschauungen autonom und existieren sie unabhängig vom Verstand, oder wenigstens können sie

9 „Ohne Sinnlichkeit würde uns kein Gegenstand gegeben und ohne Verstand keiner gedacht werden. Gedanken ohne Inhalt sind leer, Anschauungen ohne Begriffe sind blind. Daher ist es eben so nothwendig, seine Begriffe sinnlich zu machen (d.i. ihnen den Gegenstand in der Anschauung beizufügen), als seine Anschauungen sich verständlich zu machen (d.i. sie unter Begriffe zu bringen).“

10 McDowell: Mind and World, op. cit., 9.

11 Wenzel, op. cit., $414 \mathrm{f}$.

12 Vgl. McDowell: Having the World in View, op. cit., 124.

13 Ich übergehe hier das nicht unwichtige Problem, dass McDowell den Unterschied zwischen Kategorien und empirischen Begriffen herunterzuspielen scheint; vgl. Williams, op. cit., $72 \mathrm{f}$. und Connolly, Kevin: „Which Kantian Conceptualism (or Non-Conceptualism)?“ In: The Southern Journal of Philosophy 52 (3), 2014, 316-337. Selbstverständlich geht es in der Deduktion um die Frage nach der Beziehung zwischen a priori-Begriffen und Anschauungen und steht die Anwendbarkeit empirischer Begriffe nicht zur Diskussion, weswegen Griffiths (op. cit.) Kritik an Allais' nonkonzeptualistischer Position als unangemessen und sein eigener konzeptualistischer Interpretationsvorschlag als verfehlt angesehen werden muss (siehe Schulting: „Kant, Non-Conceptual Content, and the ,Second Step“ of the B-Deduction“, op. cit., 53 Fn. 5).

14 Hanna, Robert: „Kant and Nonconceptual Content“. In: European Journal of Philosophy 13 (2), 2005, 247-290, hier 259f. und ders.: „Kantian Non-Conceptualism“, op. cit., 45.

15 Allais, op. cit., $387 \mathrm{f}$. 
unabhängig vom Verstand existieren, denn sie sind in den meisten kognitiven Fällen, also in Urteilen, mit dem Verstand in einer Einheit verbunden. Dass dem so ist, scheint bestätigt zu werden durch die von Kant vertretene Ansicht, dass Tiere keinen Verstand haben, aber doch anschauen oder wahrnehmen können (z. B. V-Lo/Wiener, AA 24: 846; Log, AA 09: 65; Anth, AA 07: 196; DfS, AA 02: $59 \mathrm{f}$.) und dass sehr kleine Kinder Anschauungen haben, die unabhängig von begrifflichen Aktivitäten vorkommen (V-Anth/Collins, AA 25: 10; V-Anth/Fried, AA 25: 473). Dies bedeutet, dass mindestens in vorgenannten Fällen Anschauungen unabhängig vom Verstandesvermögen vorliegen können müssen und das Verstandesvermögen keine notwendige Bedingung des Habens von Anschauungen ist, weil man doch kaum argumentieren könne, dass sehr kleine Kinder, die noch keine Begriffe verwenden, infolgedessen überhaupt nicht wahrnähmen. ${ }^{16}$

Beide Thesen, die nonkonzeptualistische wie die konzeptualistische, beanspruchen, auf Kant zu basieren, aber wie schon gesagt, können beide jedoch nicht zugleich wahr sein. Ich möchte hier nun zwei Hauptprobleme diskutieren. Erstens möchte ich mich mit der Art und Weise befassen, in der KrV, A 89f./B 122f. von Konzeptualisten wie Nonkonzeptualisten gelesen wird; zweitens werde ich, soweit dies im gegenwärtigen Zusammenhang relevant ist, die Rolle der figürlichen Synthesis, die Kant in der zweiten Hälfte der B-Deduktion (KrV, B 151) einführt, beleuchten. Es wird sich herausstellen, dass sich der sogenannte, kantianische' Nonkonzeptualismus als weder mit dem Buchstaben noch mit dem Geist der Deduktion vereinbar erweist, da ihr Beweisziel, besonders die Begründung der auf der engen Beziehung zwischen der figürlichen Synthesis und dem Verstand beruhenden Möglichkeit raumzeitlicher Erfahrung, grundsätzlich von ihm verkannt wird. Das besagt aber keineswegs, dass der ,kantianische“ Konzeptualismus, wie er von McDowell, Pippin und anderen vertreten wird, die kantische Position widerspiegelt, da, wie wir gleich sehen werden, der Konzeptualismus Kants Verfahren in einer wichtigen Hinsicht ebenso sehr fehlinterpretiert.

16 Vgl. Allais, op. cit., 405 f. Man könnte natürlich dagegen einwenden, dass, obwohl kleine Kinder keine empirischen Begriffe verwenden und nicht urteilen, sie doch Kategorien anwenden müssen, um Objekte überhaupt erst wahrnehmen zu können; z. B. Griffith, op. cit., 207, behauptet, „even sense perception without judgment stands under the categories“. Es ist mir aber nicht einsichtig, wie Kategorien, die ja nichts anderes als Funktionen des empirischen Urteils sind (KrV, B 143), außerhalb des urteilsmäßigen Kontextes, worin empirische Begriffe allerdings verwendet werden müssen, angewandt werden könnten. 


\title{
Der Passus in KrV, A 89 f./B 122 f.: Hypothese oder reale Möglichkeit?
}

Analysieren wir zunächst die einschlägige Stelle in Paragraph 13. Diese Stelle ist ja der wichtigste Beweisgrund für die nonkonzeptualistische These, weil sie den Hauptgedanken des Nonkonzeptualismus, dass es tatsächlich Anschauungen gibt, die nicht begrifflich verarbeitet werden können müssen, zu bekräftigen scheint. Kant schreibt:

\begin{abstract}
Die Kategorien des Verstandes dagegen stellen uns gar nicht die Bedingungen vor, unter deren Gegenstände in der Anschauung gegeben werden, mithin können uns allerdings Gegenstände erscheinen, ohne daß sie sich nothwendig auf Functionen des Verstandes beziehen müssen, und dieser also die Bedingungen derselben a priori enthielte. Daher zeigt sich hier eine Schwierigkeit, die wir im Felde der Sinnlichkeit nicht antrafen, wie nämlich subjective Bedingungen des Denkens sollen objective Gültigkeit haben, d.i. Bedingungen der Möglichkeit aller Erkenntniß der Gegenstände abgeben: denn ohne Functionen des Verstandes können allerdings Erscheinungen in der Anschauung gegeben werden. [...] Denn es könnten wohl allenfalls Erscheinungen so beschaffen sein, daß der Verstand sie den Bedingungen seiner Einheit gar nicht gemäß fände, und alles so in Verwirrung läge, daß z.B. in der Reihenfolge der Erscheinungen sich nichts darböte, was eine Regel der Synthesis an die Hand gäbe [...]. Erscheinungen würden nichts destoweniger unserer Anschauung Gegenstände darbieten, denn die Anschauung bedarf der Functionen des Denkens auf keine Weise. (KrV, A 89f./B 122f.)
\end{abstract}

Dieser Passus wird meistens so gelesen, als ob Kant die Möglichkeit exorzieren wolle, dass „Erscheinungen so beschaffen [sind], daß der Verstand sie den Bedingungen seiner Einheit gar nicht gemäß fände“. Das heißt, Kants Ziel sei gerade, die Möglichkeit, dass es realiter Erscheinungen gibt, die nicht der Einheit des Verstandes gemäß sind, auszuschließen. ${ }^{17}$ Dass Anschauungen bzw. Erscheinungen nicht vom Verstande vereinigt oder verbunden seien, sondern wie „ein Gewühle“ (KrV, A 111) im völligen Durcheinander lägen, ist also dieser Lesart zufolge nur eine Hypothese, die Kant zu eliminieren beabsichtigt, nicht eine reale Möglichkeit, die er zugesteht. In der Deduktion (d.h. in der B-Auflage im ,zweiten Schritt' in B 159 f.) begründe Kant dies nämlich damit, dass alle Anschauungen, mithin alle Erscheinungen, den Kategorien gemäß bestimmt sind oder wenigstens bestimmt

17 Siehe u. a. Allison, Henry: Idealism and Freedom. Essays on Kant's Theoretical and Practical Philosophy. Cambridge 1996, 49 f.; ders.: Kant's Theory of Taste. A Reading of the ,Critique of Aesthetic Judgment‘. Cambridge 2001, 37 f. und Ginsborg, op. cit., 70 f. (näheres zu Ginsborgs Deutung bei Schulting: „Kant, Non-Conceptual Content, and the „Second Step“ of the B-Deduction“, op. cit., 83f.) 
werden können müssen. Dies bedeutete folglich, dass die Möglichkeit, dass es Erscheinungen gibt, die nicht sozusagen ,konzeptualisiert' sind, ausgeschlossen werden muss, also keine metaphysische, d. h. keine reale Möglichkeit darstellt. ${ }^{18}$ Man könnte sogar behaupten, die Unterstellung selbst, dass es so etwas wie ein ,nicht-konzeptualisierter' Bewusstseinsinhalt bzw. eine ,nicht-konzeptualisierte، Erscheinung geben könne, beruhe bereits auf der Anwendung der Kategorien als notwendige Erfahrungsbedingungen. Die These des Nonkonzeptualismus stütze sich demzufolge paradoxerweise auf die Wahrheit des Konzeptualismus.

Eine derartige radikale konzeptualistische Lesart von Kants Hauptargument wird allerdings nicht von allen Konzeptualisten geteilt, denn sie erweckt den Anschein, die epistemologische mit der ontologischen oder konstitutiven Ebene zu verwechseln. Dass wir notwendigerweise nicht ohne Begriffe Erkenntnis über etwaige nicht-begrifflichen Bewusstseinsinhalte bzw. Erscheinungen gewinnen können, impliziert keinesfalls, dass Bewusstseinsinhalte bzw. Erscheinungen notwendigerweise konzeptualisiert sein müssen. Der ,starke‘ Konzeptualismus sollte unterschieden werden vom ,schwachen' Konzeptualismus, der besagt, dass (1) Bewusstseinsinhalte, Anschauungen bzw. Erscheinungen nicht vom Verstand abhängen, was ihre Existenzweise angeht, (2) jedoch vom Verstand bestimmt werden können müssen. Das heißt, der ,schwache‘ Lesart zufolge müssen Bewusstseinsinhalte, Anschauungen bzw. Erscheinungen wenigstens konzeptualisierbar sein.

Dieser Unterschied zwischen epistemologischen und ontologischen Bedingungen bietet eine willkommene Gelegenheit zur Nuancierung der konzeptualistischen Position, die aber bei weitem noch nicht zulänglich ist. Er zeichnet sie noch immer als hauptsächlich konzeptualistisch im oben definierten Sinne aus, weil sie die wesentlich nonkonzeptualistische These, es könne ,prinzipiell‘ nichtkonzeptualisierbare Bewusstseinsinhalte, Anschauungen bzw. Erscheinungen geben, grundsätzlich verneint. Kurz gesagt, der ,schwache‘ Konzeptualismus behauptet, (1) es könne zwar de facto nicht-konzeptualisierte Bewusstseinsinhalte, Anschauungen bzw. Erscheinungen geben, da sie ja eben nicht automatisch auf einen Begriff gebracht seien, aber (2) es könne keine ,prinzipiell' nicht-konzeptualisierbaren Bewusstseininhalte usw. geben, d.h., Bewusstseinsinhalte seien ,prinzipiell‘ konzeptualisierbar. Gerade diese letztere Annahme wird von sogenannten ,essentialistischen' Nonkonzeptualisten, wie Hanna, verneint. ${ }^{19}$

18 Gomes, op. cit., 6, unterscheidet übrigens zwischen „epistemischer“ und „metaphysischer“ Möglichkeit.

19 Hanna: „Kant, Hegel, and the Fate of Non-Conceptual Content“, op. cit., 5, zufolge muss der Nonkonzeptualismus, d.h. der Inhalts-Nonkonzeptualismus, in zwei Arten unterteilt wer- 
Es gibt aber noch eine weitere Schwierigkeit. Mit der ,schwach'-konzeptualistischen These, es gebe keine ,prinzipiell' nicht-konzeptualisierbaren Anschauungen, schleicht sich heimlich doch noch eine existentielle Annahme ein, nämlich die negative existentielle Aussage, dass solche Anschauungen nicht vorliegen können. Dass wir nur begrifflichen Zugang zu Anschauungen haben, impliziert natürlich weder, dass Anschauungen sozusagen ,immer schon“ konzeptualisiert sind, noch dass sie irgendwie eine Disposition aufweisen, konzeptualisiert zu werden. Es läßt sich hier den Verdacht des konzeptuellen Idealismus aufwerfen, der beansprucht, dass alles was wir unter Begriffe bringen können, selbst schon begrifflich oder zumindest proto-begrifflich sein muss. Laut Kants Kerngedanken, dass wir von den Dingen nur a priori wissen können „was wir selbst in sie legen“ (KrV, B XVIII) - die Quintessenz synthetischer Erkenntnis a priori -, wäre es aber unmöglich die Dinge, wie sie an sich selbst sein mögen, zu erkennen oder wenigstens ihre etwaige Disposition, erkennbar zu sein, bestimmen zu können. Man kann eben nicht wissen, im strengen kantischen Sinn von objektiv gültiger Erkenntnis, dass es nicht Bewusstseinsinhalte, Anschauungen bzw. Erscheinungen geben könne, die sich grundsätzlich dem Erkenntnisvermögen entziehen, weil wir eben nur von den Dingen wissen können, was wir zuvor in sie legen. Was nicht mittels einer synthetischen a priori Begründung bewiesen werden kann, kann man im besagten Sinn auch nicht wissen.

den: der ,essentialistische‘ Inhalts-Nonkonzeptualismus besagt, dass nicht-begrifflicher Inhalt und begrifflicher Inhalt wesentlich verschieden sind, entgegen dem ,nicht-essentialistischen Inhalts-Nonkonzeptualismus, der besagt, dass nicht-begrifflicher Inhalt und begrifflicher Inhalt sich nur kontingenterweise unterscheiden. Laut des ersteren gibt es Bewusstseinsinhalte bzw. Anschauungen, die sich grundsätzlich der begrifflichen Verarbeitung (,Konzeptualisation') entziehen (das Feinkörnigkeitsargument). Die letztere Form des Nonkonzeptualismus, d.h. der ,nicht-essentialistische', konvergiert mit dem Allais'schen ,relativen' Nonkonzeptualismus; siehe oben Fußnote 7. Tolley, Clinton: „The Non-Conceptuality of the Content of Intuitions: A New Approach“. In: Kantian Review 18 (1), 2013, 107-136, hier 115, verteidigt eine Art von ,essentialistischem 'Inhalts-Nonkonzeptualismus, der besagt, dass der wesentliche Unterschied zwischen Begriffs- und Anschauungsinhalt in den unterschiedlichen Weisen liegt, in denen Begriffe und Anschauungen mittelbar bzw. unmittelbar auf den Objekt bezogen sind. Ein derartiger InhaltsNonkonzeptualismus schließe ein Verhältnis zwischen Anschauungen und Begriffen nicht aus, indem nämlich Begriffe mittels Anschauungen auf Gegenständen angewandt werden können. Im Zusammenhang einer Analyse des Arguments der A-Deduktion klassifiziert Grüne, Stefanie: Blinde Anschauung. Die Rolle von Begriffen in Kants Theorie sinnlicher Synthesis. Frankfurt a/M. 2009, vier möglichen Interpretationsvarianten des Verhältnisses zwischen Anschauung und Begriff: die „urteilstheoretische“, die „nonkonzeptualistische“, die „konzeptualistische“ und die „obskuristisch-konzeptualistische“ Variante, welch letztere ihre eigene ist. In Schulting: Kant’s Radical Subjectivism, op. cit. Kap. 6, beschäftige ich mich mit Grünes Lesart der „sinnlichen“ Synthesis in Bezug auf den Nonkonzeptualismus. 
Die Bezeichnung ,prinzipiell‘ im Ausdruck ,prinzipiell konzeptualisierbar‘ unterscheidet den ,schwachen' Konzeptualismus demnach nicht hinreichend von der Definition des ,starken“ Konzeptualismus. Denn unterstellt die These, dass Bewusstseinsinhalte, Anschauungen bzw. Erscheinungen ,prinzipiell` konzeptualisierbar seien, nicht schon die Wahrheit des ,starken' Konzeptualismus? Die real mögliche, d.h. reine, wesentliche Nonkonzeptualität von Bewusstseinsinhalten usw. wird damit nämlich letztendlich prinzipiell bestritten.

Ich möchte nun nochmals auf den Passus in KrV, A 89 f./B 122f. zurückkommen. Brady Bowman hat jüngst zu dieser Stelle folgendes geschrieben:

I read Kant's remarks here as saying that objects can, in the sense of a formal logical possibility, appear to us without necessarily having a relation to the functions of the understanding, and hence that a transcendental deduction is required in order to show why it is nevertheless really or transcendentally impossible that appearances and concepts come apart, on pain of destroying the unity of consciousness. ${ }^{20}$

Bowman unterscheidet hier zu Recht zwischen dem, was ,logisch möglich“ bzw. ,logisch unmöglich' und was ,transzendental möglich' bzw. ,transzendental unmöglich` ist. Selbstverständlich kann man auf Grund von Kants ,Zwei Grundquellen'-Theorie (KrV, A 50/B 74) die logische Möglichkeit, dass uns ein Gegenstand erscheint, ohne dass eine Beziehung zum Verstand vorliegen muss, nicht ausschließen, da Gegenstände eben nicht analytisch aus Begriffen seziert werden können. ${ }^{21}$ Erscheinungen könnten so beschaffen sein, dass sie keine Beziehung zum Verstand haben, wenn in irgendeiner möglichen, d. h. hypothetischen Welt, das Bestehen der Dinge nicht der Einheit des Verstandes gemäß sein sollte. Bowman räumt diese Möglichkeit ein, schließt jedoch die reale oder, was er selbst die „transzendentale“ Möglichkeit ${ }^{22}$ eines derartigen Zustandes nennt, aus.

20 Bowman, Brady: „A Conceptualist Reply to Hanna’s Kantian Non-Conceptualism“. In: International Journal of Philosophical Studies 19 (3), 2011, 417-446, hier 422.

21 Für Leibniz erscheinen Objekte in der Sinnlichkeit nicht ohne implizit eine Beziehung zum Verstand zu haben, da der Unterschied zwischen der Wahrnehmung eines Gegenstandes und seines Gedachtwerdens lediglich einer zwischen mehr oder weniger Klarheit ist. Für Kant dagegen kann die Wahrnehmung eines Gegenstandes nicht aus seinem Begriff analysiert werden, denn der Unterschied zwischen sinnlicher Wahrnehmung und dem Begriff ist ein Unterschied der Art nach. Immerhin beansprucht Kant, dass es eine notwendige Beziehung, die allerdings nicht eine analytische ist, zwischen der Wahrnehmung eines Gegenstandes und seinem Begriff gibt. In der Deduktion gilt es diese Beziehung, die Kant als ,synthetisch a priori“ bezeichnet, aufzuzeigen.

22 Allison, Henry: Essays on Kant. Oxford 2012, 48, nennt dies dasjenige, was „phänomenologisch“ möglich wäre. Das heißt, Allison behauptet, es sei „phänomenologisch“ unmöglich sich 
Aber behauptet Kant hier in KrV, A 89f./B 122f. in der Tat, es sei realiter unmöglich, dass Erscheinungen und die einheitsstiftenden Funktionen des Verstandes auseinandertreten? Bowman betont ausdrücklich, Kants Argumentation müsse hier explizit kontrafaktisch gelesen werden. ${ }^{23}$ Obschon Kant kontrafaktisch zu formulieren und somit nur auf eine ,logisch mögliche“ Nichtübereinstimmung anzuspielen scheint, glaube ich allerdings nicht, dass die kontrafaktische Deutung in Einklang damit gebracht werden kann, dass Kant einen ausdrücklichen Beweggrund für seine Argumentation angibt, der nicht als lediglich hypothetisch gedeutet werden kann, nämlich das Faktum, dass „die Anschauung [...] der Functionen des Denkens auf keine Weise [bedarf]“ (KrV, B 123/A 91). Das heißt, die ,Zwei Grundquellen'-Theorie wirft aufgrund der realen Kluft zwischen Sinnlichkeit und Verstand und der Tatsache, dass ihre Funktionen nicht vertauscht werden können (KrV, B 75/A 51), das wirkliche, metaphysische Problem auf, das Kant mit der ,Transzendentalen Deduktion' zu lösen beabsichtigt. ${ }^{24}$

Die Nichtübereinstimmung zwischen den Erscheinungen und dem Verstand betrifft nicht eine logische oder epistemische Möglichkeit, die schließlich als eine nicht-reale erwiesen werden soll, sondern ein echtes Problem, das sich aus Kants Ablehnung des Leibniz'schen-Wolffianischen Vertrauens in die grundsätzlich prästabilierte Übereinstimmung zwischen Sinnlichkeit und Verstand ergibt. Kants Anliegen in der Deduktion ist daher vielmehr aufzuzeigen, dass obgleich es eine reale Möglichkeit gibt, dass sich Erscheinungen in unserer Anschauung anbieten, ohne dabei mit dem Verstand in einer Einheit verbunden zu sein, nachgewiesen werden kann, dass, wenn und nur wenn wir mittels unseres Urteilsver-

von Anschauungen, die sich nicht nach unserem Verstand richten, bewusst zu sein. Oben habe ich angedeutet, dass es sich um die metaphysische, was Bowman „transzendentale“, im Gegensatz zur logischen oder „epistemischen“ (Gomes, op. cit., 6) Möglichkeit handelt. Die „phänomenologische“ Unmöglichkeit, wovon Allison redet, schließt die metaphysische Möglichkeit eines Auseinandertretens der Anschauungen und des Verstandes übrigens nicht aus.

23 Bowman, op. cit., 423. Grüne, Stefanie: „Is there a Gap in Kant’s B Deduction?“ In: International Journal of Philosophical Studies 19 (3), 2011, 465-490, hier 475 f., behauptet, der Passus in KrV, A 89f./B 122f. habe nur eine „didaktische Funktion“ und der Schluss der Deduktion schließe letztendlich aus, dass Anschauungen gegeben werden können, ohne auf die Funktionen des Denkens bezogen zu sein. Laut Grüne möchte Kant in diesem einleitenden Paragraphen nur motivierend erläutern, worum es in einer Deduktion gehen soll.

24 Gomes (op. cit., 6) behauptet hingegen, „we are not forced to treat the possibility expressed at A 90/B 123 as metaphysical“, d.h. als real möglich; das indikativische ,können“ in KrV, B 122 sei vereinbar mit ,the three paragraphs which end that section (A 89-92/B 122-124) operating under an assumed ,for all we know` operator“. In dem von Gomes erwähnten Textteil (KrV, B 123) verwendet Kant den Konjunktiv. 
mögens Erscheinungen als Gegenstände für unseren Verstand apprehendieren, sie auf letzteren bezogen sein müssen.

Kant nimmt in der Deduktion keineswegs wieder zurück, was er vorher, in der Einleitung zur ,Transzendentalen Logik` bekräftigt hat, nämlich die absolute Trennung von Sinnlichkeit und Verstand. Statt die Kluft zwischen Anschauung und Begriff zu überbrücken, wie Bowman und andere Konzeptualisten zu glauben scheinen, will Kant vielmehr zeigen, wie aus deren Vereinigung erst Erkenntnis entspringt (KrV, B 75/A 51). Nichts Wesentliches an Anschauungen oder Erscheinungen bewirkt, dass sie sich irgendwie an den Verstand anknüpfen oder dass sie eine derartige Disposition aufweisen, dass sich der Verstand an sie anknüpft. Meines Erachtens ist gerade diese reale Kluft das einschlägige Anfangsproblem für Kant. Kant zeigt, dass wenn und nur wenn Anschauungen und damit die Erscheinungen kognitiv relevant für uns sein sollen, Anschauungen notwendigerweise auf die Funktionen des Denkens bezogen sind. Das heißt, Anschauungen werden nur in dem Fall von den einheitsstiftenden Funktionen des Verstandes bestimmt, in dem der Verstand sie als von ihm bestimmt apprehendiert. Der Verstand kann ja nur bestimmen, was er selbst als von ihm bestimmbar apprehendiert. Es kann realiter, etwa bei kognitiver Störungen oder in der Tat bei kleinen Kindern und Tieren, also Anschauungen geben, die nicht vom Verstand apprehendiert werden und deshalb nicht zur Erkenntnis (im strengen kantischen Sinne) beitragen.

Es lässt sich vermuten, dass Konzeptualisten wie Bowman die Bezeichnung $<$ alle Erscheinungen> verwechseln mit <alle Objekte der möglichen Erfahrung>. ${ }^{25}$ Aufgrund von Kants Theorie der Erfahrung ist es analytisch wahr zu sagen, es gelte notwendigerweise für jedes mögliche Objekt der Erfahrung, dass es durch die Kategorien vom Verstand bestimmbar sei, da ja ein Objekt per definitionem eine aufgrund der Kategorien bestimmte Einheit des Bewusstseins ist und die Kategorien aus dieser Einheit ableitbar sind. ${ }^{26}$ Aber Konzeptualisten versäumen meistens zu erwähnen, dass die termini $<$ Erscheinung $>$ und $<$ Objekt $>$ im Sinne von $\mathrm{KrV}$, B 137 („das, in dessen Begriff das Mannigfaltige einer gegebenen Anschauung vereinigt ist") nicht bedeutungsgleich sind. ${ }^{27}<$ Erscheinung $>$ ist laut Kants Definition am Anfang der ,Transzendentalen Ästhetik“ der „unbestimmmte“ Ge-

25 Connolly, op. cit., begeht denselben Fehler.

26 Siehe Schulting, Dennis: Kant's Deduction and Apperception. Explaining the Categories. Basingstoke und New York 2012.

27 Williams, op. cit., 69, weist ausnahmsweise explizit auf den hier genannten Unterschied zwischen $<$ Erscheinung $>$ und $<$ Objekt $>$ hin, aber unterlässt, ihre wesentliche Differenz näher auszuführen. 
genstand einer empirischen Anschauung (KrV, B 34/A 20), also ein Mannigfaltiges einer noch nicht vereinigten Anschauung. ${ }^{28}$ Eine Erscheinung, als eine noch nicht vereinigte Anschauung, ist folglich nicht schon von selbst objektiv gültig, entgegen der Meinung von Nonkonzeptualisten wie Hanna ${ }^{29}$ und Allais ${ }^{30}$, die behaupten, dass eine Anschauung schon durch sich selbst, also intrinsisch, objektiv referiere (siehe weiter unten).

Die Klasse der Erscheinungen ist demnach viel größer als die Klasse der Objekte möglicher Erfahrung. Die erstere enthält nicht nur alle möglichen Erfahrungsobjekte, sondern auch alle solche Erscheinungen, die nur subjektive Gültigkeit haben und niemals zur Objektivität gelangen, ,Praussische‘ subjektive Objekte etwa, ${ }^{31}$ welche als solche nicht zur letzteren Klasse gehören. ${ }^{32}$ Nicht alle Erscheinungen sind somit schon von Anfang an, als „bloße“ empirische Vorstellungen oder „Vorstellungsarten“ (KrV, A 372; vgl. B 242 f./A 197 f.), auf den Verstand bezogen, wie der Konzeptualist meint.

Aber wäre dies Grund zur Annahme der nonkonzeptualistischen These, nämlich dass wir eine Anschauung im Sinne einer objektiven Perzeption eines bestimmten Gegenstandes ohne Mitwirkung des Verstandes haben können? Wie ich schon erwähnt habe, glauben Nonkonzeptualisten wie Hanna und Allais, basierend auf Kants Singularitätskriterium (KrV, A 320/B 37733; A 68/B 93; A 19/B 33; B 47/A 3234), eine Anschauung referiere bereits objektiv und sei somit objektiv gültig, unabhängig vom Verstand und dessen einheitsstiftender, auf ein tatsäch-

28 In der A-Deduktion, in KrV, A $108 \mathrm{f}$., erläutert Kant das Verhältnis zwischen Erscheinung und Anschauung folgendermaßen: „Erscheinungen sind die einzigen Gegenstände, die uns unmittelbar gegeben werden können, und das, was sich darin unmittelbar auf den Gegenstand bezieht, heißt Anschauung. Nun sind aber diese Erscheinungen nicht Dinge an sich selbst, sondern selbst nur Vorstellungen [...].“

29 Hanna: „Kant and Nonconceptual Content“, op. cit., 258; ders.: „Kantian Non-Conceptualism“, op. cit., 50 f. und ders.: „The Togetherness Principle, Kant’s Conceptualism, and Kant's Non-Conceptualism“, op. cit.

30 Allais, op. cit., $391 \mathrm{f}$.

31 Vgl. Prauss, Gerold: Erscheinung bei Kant. Ein Problem der ,Kritik der reinen Vernunft'. Berlin 1971, $16 \mathrm{f}$.

32 Vgl. KrV, A 107, wo Kant vom „Flusse innrer Erscheinungen“ spricht.

33 ,[Eine] Anschauung [...] bezieht sich unmittelbar auf den Gegenstand und ist einzeln.“

34 „Die Vorstellung, die nur durch einen einzigen Gegenstand gegeben werden kann, ist aber Anschauung.“ Laut Rohs, Peter: „Bezieht sich nach Kant die Anschauung unmittelbar auf Gegenstände?“ In: Kant und die Berliner Aufklärung. Akten des 9. Internationalen Kant-Kongresses, Bd. II. Hrsg. von V. Gerhardt, R.-P. Horstmann und R. Schumacher. Berlin und New York 2001, 214-228, kann man dies als einen „rein singulären Gegenstandsbezug“ bezeichnen (zitiert von Wenzel, op. cit., 409). Siehe auch V-Met/Schön, AA 28: 484 und FM, AA 20: 325. 
liches Urteil hinauslaufenden Wirkung. Aber dies kann schon deswegen nicht der Fall sein, weil, obwohl eine Anschauung eine unmittelbare Vorstellung des gegebenen Gegenstandes ist (V-Met/Schön, AA 28: 484) und somit in gewisser Hinsicht vom gegebenen Gegenstand abhängig ist (KrV, B 72), objektive Gültigkeit, welche erst die Gegenständlichkeit des Gegenstandes konstituiert, für Kant streng genommen eine alleinige Funktion des Urteils ist (KrV, B $141 \mathrm{f}.){ }^{35}$

\section{Der Nonkonzeptualismus und die figürliche Synthesis in der B-Deduktion}

Ein weiterer, schwerwiegender Grund, weshalb der ,kantianische‘ Nonkonzeptualismus letztendlich nicht haltbar ist - obwohl er zu Recht gegen die konzeptualistische These darauf hinweist, dass nicht alle Erscheinungen, entweder explizit oder implizit, auf den Verstand bezogen sein müssen -, betrifft die Rolle der sogenannten figürlichen Synthesis oder synthesis speciosa. Nonkonzeptualisten wie Hanna und Allais halten es für unfraglich, dass diejenigen Anschauungen bzw. Erscheinungen, die nicht auf den Verstand bezogen sind, nichtsdestoweniger nicht nur, wie gesagt, objektiv gültig sind und infolgedessen objektiv referieren, sondern auch schon aufgrund der figürlichen Synthesis, welche formell von der intellektuellen Synthesis unterschieden werden muss (KrV, B $151 \mathrm{f}$.), synthetisiert sind. Zweitens betonen sie, dass obwohl ein Urteil Synthesis voraussetze, Synthesis nicht ebenso ein Urteil oder wenigstens begriffliche Verarbeitung (,Konzeptualisation') oder Begrifflichkeit voraussetze. ${ }^{36}$ Das Verhältnis sei nicht reziprok. Mit anderen Worten: Nicht alle Formen der Synthesis setzten den Verstand, und damit den Kategorien, voraus. Die Ansicht, Synthesis involviere nicht notwendigerweise Begriffe, ist aber schon deswegen problematisch, weil Kant zufolge die transzendentale Synthesis der Einbildungskraft, wovon ja im Kontext der Debatte die Rede ist, eng verbunden ist mit der Einheit der Apperzeption, wodurch „ein Begrif vom Gegenstande überhaupt gedacht [wird] nach den verschiedenen Arten der transscendentalen Synthesis“ (LBl KrV-I, B 12, AA 23: 18). Die transzendentale

35 Es sei jedoch zu bemerken, dass Allais, op. cit., 405, 407, 413, einen wichtigen Unterschied macht zwischen dem, was sie das „Präsentiertwerden“ von Objekten nennt, wofür die Anschauung einzig und allein verantwortlich sei, und die Vorstellung des Objektes als Objekt, was die Kategorien und damit das Urteilsvermögen voraussetzt.

36 Siehe insbesondere Allais, op. cit., 396. 
Synthesis der Einbildungskraft, oder die figürliche Synthesis, setzt demnach mindestens einen Begriff, nämlich den vom Gegenstand überhaupt, voraus. ${ }^{37}$

Der entscheidende Irrtum betrifft aber die nonkonzeptualistische Auslegung des Verhältnisses zwischen der intellektuellen und figürlichen Synthesis. Da Hanna $^{38}$ und Allais ${ }^{39}$ die Ansicht vertreten, dass die figürliche Synthesis oder die Synthesis der Einbildungskraft die vom Verstand unabhängigen Anschauungen schon intrinsisch kennzeichnet, müssen sie die figürliche Synthesis von der intellektuellen gleichsam abkoppeln, da die intellektuelle Synthesis offensichtlich eine „Verstandesverbindung“ ist (KrV, B 151). Eine solche Trennung von intellektueller und figürlicher Synthesis verkennt jedoch, dass Kants Vorgehensweise in der Deduktion der Art ist, dass das Argument des ,zweiten Schrittes', welches die Möglichkeit der Wahrnehmung von raumzeitlich bestimmten Objekten betrifft, ein Teilargument einer regressiven Analyse der konstitutiven Elemente möglicher Erfahrung ist. Obwohl der ,zweite Schritt“ eine in gewisser Hinsicht vom ersten Teilargument (§§15-20) unabhängige Beweisführung umfasst, ist die Abfolge der Argumentation insgesamt offensichtlich von der im ,ersten Schritt‘ erörterten intellektuellen Synthesis zur figürlichen im zweiten und nicht umgekehrt. ${ }^{40}$ Die intellektuelle Synthesis ist eine notwendige Bedingung der figürlichen, während die figürliche keine notwendige Bedingung der intellektuellen Synthesis ist. Folglich kann es keine figürliche Synthesis geben, die nicht auch eine intellektuelle voraussetzte.

Da Hanna und Allais meinen, die figürliche Synthesis sei eine quasi-intrinsische Eigenschaft der Anschauung, welche sich erst in zweiter Linie möglicherweise zu einem von der intellektuellen Synthesis bewirkten Urteil entfalte, wenn es sich nämlich tatsächlich um eine urteilsmäßige Erkenntnisaussage handle also eine begrifflich artikulierte Aussage, die laut Hanna erst aufbaue auf der figürlichen Synthesis ${ }^{41}$-, wird das Bedingungsverhältnis der intellektuellen und figürlichen Synthesis verkehrt. Hanna meint sogar, dass die letztere der „,notwen-

37 Man könnte sich natürlich aber auch ausschließlich auf die A-Auflage der Kritik berufen, wie Allais, op. cit., 396, 396 f.n.37, es mit Verweis auf KrV, A 77 f./B 103, A 98-102 und A 115 f. tatsächlich tut, und auf dieser Basis behaupten, nur die Synthesis der Rekognition, und gerade nicht die Synthesis der Apprehension und der Reproduktion, involviere begriffliche Verarbeitung. Zur Kritik einer dergleichen Position vgl. Schulting: Kant's Radical Subjectivism, op. cit., Kap. 6.

38 Hanna: „Kant and Nonconceptual Content“, op. cit., 249; ders.: „Kantian Non-Conceptualism“, op. cit., 62.

39 Allais, op. cit., $394 \mathrm{f}$.

40 Vgl. Allison: Essays on Kant, op. cit., $35 \mathrm{f}$.

41 Vgl. Hanna: „Kantian Non-Conceptualism“, op. cit., 45 f., 58 f. 
dige Grund“ der ersteren Synthesis sei und ihre eigene nicht-reduzierbare „niedrigere“ Spontaneität habe. ${ }^{42}$

Wie gesagt, halten Hanna und Allais es für möglich, dass eine figürlich synthetisierte Anschauung vorliegt, ohne dass die Bedingungen der intellektuellen Synthesis, also die Bedingungen des Verstandes, erfüllt worden sind. In der kantischen Reihenfolge kann formaliter tatsächlich eine intellektuelle Synthesis ohne figürliche Synthesis vorliegen, ${ }^{43}$ aber eine vorliegende figürliche Synthesis impliziert immer schon eine intellektuelle, weil die figürliche Synthesis gerade eine spezifischere Form der intellektuellen Synthesis ist. Genauer gesagt, die figürliche Synthesis ist, wie Kant in $\S 24$ (KrV, B 152) zu erkennen gibt, „eine Wirkung des Verstandes auf die Sinnlichkeit und die erste Anwendung desselben [...] auf Gegenstände der uns möglichen Anschauung“ (Hervorhebung von mir). Die figürliche Synthesis ist ebenso sehr eine Aktivität, die vom Verstande ausgeübt wird wie die intellektuelle Synthesis, welch letztere als „Verstandesverbindung“ freilich nur ,in Ansehung des Mannigfaltigen einer Anschauung überhaupt in der bloßen Kategorie gedacht“ wird (KrV, B 151). Kurz, figürliche und intellektuelle Synthese sind nur in der formalen Analyse, in der ,Transzendentalen Deduktion“ also, aber nicht in Wirklichkeit, in der Erfahrung oder in einem Urteil, zu trennen.

Die nonkonzeptualistische These einer von der begrifflichen Verstandeshandlung streng zu unterscheidenden figürlichen Synthesis verstößt auch gegen den Geist des Leitfadens, der in nuce ankündigt, was Kant in der ,Transzendentalen Deduktion' beweisen wird, nämlich den Gedanken von einem engen Zusammenhang zwischen den beiden formal unterschiedlichen Einheitsfunktionen desselben Verstandes, der sowohl ,vermittelst der synthetischen Einheit des Mannigfaltigen in der Anschauung überhaupt in seine Vorstellungen einen transscendentalen Inhalt [bringt]“ als „,in Begriffen vermittelst der analytischen Einheit die logische Form eines Urtheils zu Stande“ bringt (KrV, A 79/B 105). ${ }^{44}$ McDowell zufolge darf man sagen, dass die Einheit, welche von der produktiven Einbildungskraft - die ja diese einheitsstiftende Funktion des Verstandes im Feld der Sinnlichkeit ist - bewirkt wird, nicht „ein Amalgam [...] von Bauteilen, welche gesondert zur Sinnlichkeit und zum Verstand gehören“"45, sei. Die Synthesis der Einbildungskraft in der Sinnlichkeit ist keine andere als die intellek-

42 Hanna: „Kantian Non-Conceptualism“, op. cit., 62. Für eine Kritik dieser Position siehe Schulting: „Kant, Non-Conceptual Content, and the ,Second Step“ of the B-Deduction“, op. cit., 68-73. 43 Z.B. eine kategoriale Synthesis, die eine sinnliche nicht-menschliche Anschauung voraussetzte (vgl. KrV, B 148).

44 Näheres zu diesem Thema bei Schulting: Kant's Deduction and Apperception, op. cit., 83-90. 45 McDowell: Having the World in View, op. cit., 124. 
tuelle Synthesis im Feld der Sinnlichkeit, und zwar genau dann, wenn es sich um ein faktisches, empirisches Urteil handelt. Sie sind beide Aspekte derselben ursprünglichen a priori Synthesis, die der Grund unserer Erkenntnis im Urteil ist. Wie Kant in KrV, B 152 sagt, ist die figürliche Synthesis, oder die transzendentale Synthesis der Einbildungskraft, eine „Anwendung“ des Verstandes und somit der intellektuellen Synthesis (da die „Verstandesverbindung“, abstrahiert von der empirischen Anschauung, lediglich die „synthesis intellectualis“ ist) ${ }^{46}$. Wenn die figürliche Synthesis eine „Anwendung“ des Verstandes ist, dann ist eine figürliche Synthesis, die unabhängig vom Verstand sein soll und infolgedessen außerhalb des Urteils vorliege, selbstverständlich schlicht unmöglich. ${ }^{47}$

Zum Schluss möchte ich noch auf eine letzte, eher systematische Schwierigkeit hinweisen. Die nonkonzeptualistische Behauptung, die figürliche Synthesis müsse aufgrund der Unabhängigkeit von Anschauungen als getrennt von der intellektuellen Synthesis aufgefasst werden, führt zu einem unendlichen Regress. Synthesis a priori - und man sei daran erinnert, dass auch die figürliche Synthesis eine transzendentale a priori Synthesis ist (KrV, B 151) - ist gerade deswegen von Kant konzipiert worden, damit ein unendlicher Regress vermieden werde. Könnten die figürliche und intellektuelle Synthesis realiter getrennt werden und könnten sie ihre Funktion somit gesondert, im Falle der Anschauung gerade außerhalb eines Urteils, ausüben, wie ,kantianische‘ Nonkonzeptualisten wie Hanna und Allais behaupten, dann fragt man sich, welche Synthesis deren Synthesis ermöglichen soll, wenn es sich um ein tatsächliches Urteil handelt? Es gibt ja keine originärere Synthesis als die ursprünglich-synthetische Einheit der Apperzeption, die ihre Anwendung in der Sinnlichkeit in Gestalt der transzendentalen Einbildungskraft hat.

In einem neueren Beitrag antwortet Hanna auf diese $\mathrm{Kritik}^{48}$, die ich schon in einem früheren Aufsatz geäußert hatte ${ }^{49}$. Er gesteht ein, dass die oben erwähnte Problematik eines Regresses, welche er als „the schmimagination vicious regress

46 Siehe auch KrV, B 154, wo Kant „die transscendentale Handlung der Einbildungskraft“ mit dem „synthetische[n] Einfluß des Verstandes auf den inneren Sinn“ identifiziert.

47 Vgl. KrV, B 164, wo Kant bestätigt, dass, was die Einheit der intellektuellen Synthesis der sinnlichen Anschauung betrifft, die Einbildungskraft „vom Verstande [...] abhängt“ (Hervorhebung von mir). Näheres zum Thema ,figürliche Synthesis‘ in Bezug auf die Beweisführung des ,zweiten Schrittes ' der B-Deduktion bei Schulting: Kant’s Radical Subjectivism, op. cit., Kap. 7.

48 Hanna: „The Togetherness Principle, Kant's Conceptualism, and Kant’s Non-Conceptualism“, op. cit.

49 Schulting: „Kant, Non-Conceptual Content, and the ,Second Step“ of the B-Deduction“, op. cit., $70 \mathrm{f}$. 
problem" bezeichnet, eine der zwei fundamentalen Schwierigkeiten in der Debatte bezüglich Kants mutmaßlichen Nonkonzeptualismus bzw. Konzeptualismus ist. Es bleibt jedoch unklar, wie Hanna diese Schwierigkeiten beseitigen möchte. Er betont, beinahe entschuldigend, dass die Textbelege zweideutig seien und sowohl die nonkonzeptualistische als die konzeptualistische These stützten und dass uns nur eine größere Einsicht in die Art und Weise, in der beide Thesen sich auf Interpretationen der kantischen Philosophie insgesamt auswirken, weiterbringen könne.

Aufgrund der oben erläuterten interpretativen und systematischen Schwierigkeiten glaube ich allerdings eher nicht, dass die Nonkonzeptualismus-Debatte in irgendeiner Form gewinnbringend herangezogen werden kann, um einen tieferen Einblick in die Deduktion zu erhalten. Dies scheint letztlich auch Hanna selbst zuzugestehen, wenn er sagt, Kants ,essentialistischer' Nonkonzeptualismus stehe im klaren Widerstreit mit dem Hauptanspruch der ,Transzendentale Deduktion' und führe, anstatt die Beziehung zwischen Anschauung und Begriff grundlegend zu erklären, vielmehr zu einem „logical gap“, womit sich Kants Theorie als ein „deeply conflicted Conceptualism“ erweise. ${ }^{50}$ Das heißt, laut Hanna wird Kants Deduktionsprojekt gekennzeichnet durch ein fast unlösbares Dilemma, das er als das andere fundamentale Problem in der Nonkonzeptualismus-Debatte bezeichnet: entweder (a) der ,kantianische“ Nonkonzeptualismus ist wahr, und es gibt unabhängig vom Verstand objektiv gültige Anschauungen, was aber dann im Widerstreit mit Kants Hauptanliegen in der Deduktion stünde, nämlich zu beweisen, dass alle objektiv gültigen Anschauungen dem Verstandesvermögen unterliegen; die Kluft, von der Hanna spricht, wäre dann die Nichtübereinstimmung der „,cognitive scope[s]“51 von Sinnlichkeit bzw. Verstand; oder aber (b) der ,kantianische‘ Konzeptualist hat Recht, weil die Bereiche der Sinnlichkeit bzw. des Verstandes sich genau überschneiden und es daher keine Kluft (im Sinne Hannas) gibt, was aber Kants Zugeständnis widerspricht, wonach es Anschauungen ohne die Funktionen des Verstandes oder Kategorien geben kann. Dieses Dilemma rührt meines Erachtens aber nicht von einem inhärenten, tiefen Konflikt in der ,Transzendentalen Deduktion“ her, sondern ist die unmittelbare

50 Hanna: „Kant, Hegel, and the Fate of Non-Conceptual Content“, op. cit., 6. Siehe auch Hanna, Robert: „Kant’s Non-Conceptualism, Rogue Objects, and the Gap in the B Deduction“. In: International Journal of Philosophical Studies 19 (3), 2011, 397-413. In Schulting: Kant's Deduction and Apperception, op. cit., 50-61, untermauere ich mit Argumenten den Standpunkt, dass es kein „logical gap“ im Sinne von Hannas Kritik in der B-Deduktion gibt.

51 Hanna: „The Togetherness Principle, Kant’s Conceptualism, and Kant’s Non-Conceptualism“, op. cit. 
Folge eines von außen herangetragenen, wesentlich kantfremden konzeptuellen Schemas, das von vornherein quer zu Kants Anliegen steht.

Dem Nonkonzeptualisten muss jedoch eingeräumt werden, dass man auf Grund von Kants Argumenten in der Deduktion, besonders dem erörterten Passus in KrV, A 89f./B 122f., berechtigt ist zu sagen, wie der Nonkonzeptualist ebenfalls meint, es gebe tatsächlich Anschauungen, welche nicht nur de facto nicht begrifflich verarbeitet sind (dies könnte auch der Konzeptualist zugestehen), sondern auch nicht einmal begrifflich verarbeitet werden können müssen (was der Konzeptualist grundsätzlich verneint). Dies ist aber nur zutreffend unter dem Vorbehalt, dass man berücksichtigt, dass solche Anschauungen eo ipso (1) nicht synthetisiert sind (weder von der intellektuellen, noch von der figürlichen Synthesis), (2) demzufolge nur gemäß psychologischen Gesetzen im menschlichen Geist veranlasste Aggregate von Vorstellungen oder Erscheinungen sind, also im transzendentalen Sinn „bloß Mannigfaltiges“ geben (KrV, B 160 Fn.), (3) obwohl ein unmittelbar raumzeitlich gegebener Gegenstand ihnen laut Definition zugrunde liegt, keine bestimmte Wahrnehmungen räumlich unterschiedener Objekte sind und folglich (4) nicht objektiv referieren im eigentlichen kantischen Sinne. Kant kann deshalb vielleicht nur noch im trivialen Sinn ein Nonkonzeptualist genannt werden, nämlich in dem Sinne, dass Anschauungen als solche seiner ,Zwei Grundquellen'-Theorie zufolge nun einmal keine Begriffe sind und deswegen auch nicht, prinzipiell‘ konzeptualisierbar sein müssen. 